\title{
MUTATIONS IN THE CYSTIC FIBROSIS GENE IN PATIENTS WITH CONGENITAL ABSENCE OF THE VAS DEFERENS
}

\author{
Miguel Ghillón, Ph.D., Teresa Gasals, B.S., Bernard Mercier, Ph.D., Lluís Bassas, M.D., \\ Willy Lissens, Ph.D., Sherman Silber, M.D., Marie-Gatherine Romey, B.S., Javier Ruiz-Romero, M.D., \\ Glaudine Verlingue, M.D., Mireille Glaustres, M.D., Ph.D., Virginia Nunes, Ph.D., \\ Glaude Férec, M.D., Ph.D., and Xavier Estivill, M.D., Ph.D.
}

\begin{abstract}
Background. Congenital bilateral absence of the vas deferens (CBAVD) is a form of male infertility in which mutations in the cystic fibrosis transmembrane conductance regulator (CFTR) gene have been identified. The molecular basis of CBAVD is not completely understood. Although patients with cystic fibrosis have mutations in both copies of the CFTR gene, most patients with CBAVD have mutations in only one copy of the gene.

Methods. To investigate CBAVD at the molecular level, we have characterized the mutations in the CFTR gene in 102 patients with this condition. None had clinical manifestations of cystic fibrosis. We also analyzed a DNA variant (the $5 T$ allele) in a noncoding region of CFTR that causes reduced levels of the normal CFTR protein. Parents of patients with cystic fibrosis, patients with types of infertility other than CBAVD, and normal subjects were studied as controls.
\end{abstract}

$\mathrm{C}$ ONGENITAL bilateral absence of the vas deferens 1 (CBAVD) accounts for at least 6 percent of cases of obstructive azoospermia and is responsible for 1 to 2 percent of cases of infertility in men. ${ }^{1}$ CBAVD is also present in about 95 percent of male patients with cystic fibrosis, a disorder characterized by chronic pulmonary disease, pancreatic exocrine insufficiency, and elevated concentrations of electrolytes in sweat. ${ }^{2}$

Mutations in the cystic fibrosis transmembrane conductance regulator (CFTR) gene, which encodes a cyclic AMP-regulated chloride channel, ${ }^{3,4}$ have been found in patients with cystic fibrosis. ${ }^{5,6}$ Patients with the classic form of cystic fibrosis have severe mutations in each copy of the CFTR gene, whereas patients with a less severe phenotype (i.e., with normal pancreatic function and mild lung disease) have a severe mutation in one copy of CFTR and a mild mutation in the other, or mild mutations in both copies. ${ }^{7}$

Mutations in the CFTR gene have also been identified in patients with CBAVD, which suggests that this condition is a primarily genital form of cystic fibrosis. ${ }^{8-12}$ Thus, patients with CBAVD would be expected, like all patients with cystic fibrosis, to have two CFTR

From the Cancer Research Institute, Molecular Genetics Department, Hospital Duran i Reynals, L'Hospitalet de Llobregat, Barcelona, Spain (M. Chillón, T.C., V.N., X.E.); the Centre de Biogénétique, University Hospital, Brest, France (B.M., C.V., C.F.); the Andrology Department, Institute of Urology, Nephrology, and Andrology, Fundació Puigvert, Barcelona (L.B., J.R.-R.); the Department of Medical Genetics, Vrije Universiteit, Brussels, Belgium (W.L.); the Department of Urology and Microsurgery, St. Luke's Hospital, St. Louis (S.S.); the Laboratoire de Biochimie Génétique, Institut de Biologie, Montpellier, France (M.-C.R., M. Claustres); and the Genetics Service, Hospital Clinic, Barcelona (X.E.). Address reprint requests to Dr. Estivill at the Cancer Research Institute, Hospital Duran i Reynals, Avia. Castelldefels Km 2.7, 08907 L'Hospitalet de Llobregat, Barcelona, Catalonia, Spain.

Supported by a grant (93/0202E) from the Fondo de Investigaciones Sanitarias de la Seguridad Social and by grants from the Institut Català de la Salut (Generalitat de Catalunya) and the Association Française de Lutte contre la Mucoviscidose.
Results. Nineteen of the 102 patients with CBAVD had mutations in both copies of the CFTR gene, and none of them had the $5 T$ allele. Fifty-four patients had a mutation in one copy of CFTR, and 34 of them (63 percent) had the $5 T$ allele in the other CFTR gene. In 29 patients no CFTR mutations were found, but 7 of them (24 percent) had the $5 T$ allele. In contrast, the frequency of this allele in the general population was about 5 percent.

Conclusions. Most patients with CBAVD have mutations in the CFTR gene. The combination of the $5 T$ allele in one copy of the CFTR gene with a cystic fibrosis mutation in the other copy is the most common cause of CBAVD. The $5 T$ allele mutation has a wide range of clinical presentations, occurring in patients with CBAVD or moderate forms of cystic fibrosis and in fertile men. (N Engl J Med 1995;332:1475-80.)

mutations. However, few patients with GBAVD have mutations in both copies of the CFTR gene; in the majority of cases, only one mutation has been found, and in about a third no mutations have been detected. The inability of investigators to identify two CFTR mutations in these patients, even after analyzing the entire coding sequence, is not well understood, but it could be explained by the presence of mutations in noncoding regions of the gene. Such mutations would produce abnormally low levels of CFTR protein, which may cause obstruction of the vas deferens, but there may be sufficient protein to prevent disease in other organs normally affected by cystic fibrosis.

Low levels of the GFTR protein could be due to a decreased proportion of the normal messenger RNA (mRNA) of CFTR. Studies of CFTR mRNA in tissue from normal persons have identified various mRNA molecules that lack exon 4,9 , or $12 .{ }^{13-17}$ Whether or not CFTR mRNA contains exon 9 depends on the variable length of a DNA sequence of thymines in intron 8 of CFTR (Fig. 1). ${ }^{18}$ This sequence, known as a polyT sequence, contains five, seven, or nine thymines (the $5 T$, $7 T$, and $9 T$ alleles, respectively). Since the $5 T$ allele causes reduced levels of normal CFTR mRNA, ${ }^{18}$ this DNA variant would appear likely to be involved in the pathogenesis of CBAVD.

To understand the molecular genetics of CBAVD better, we have characterized the CFTR mutations in patients with this condition and studied the putative involvement of the $5 T$ allele in CBAVD and other types of male infertility.

\section{MethodS}

\section{Patients}

We studied 102 unrelated men with azoospermia and CBAVD, as diagnosed on the basis of scrotal exploration and analysis of semen 
(volume and $\mathrm{pH}$ of semen, sperm count, and concentrations of fructose and citrate). The patients came from Belgium, France, Spain, and the United States. ${ }^{2,19,20}$ None had pulmonary or gastrointestinal manifestations of cystic fibrosis. The results of sweat chloride analysis and additional clinical data on these patients have already been presented. ${ }^{12,19,20}$ The diagnoses of CBAVD were initially suggested by the clinical observation of impalpable vasa in the patients and were subsequently confirmed by analyses of semen and transrectal and abdominal ultrasonography. Each patient had a sperm count of zero.

\section{Control Subjects}

We studied 186 fathers and 44 mothers of patients with cystic fibrosis, each of whom carried a known CFTR mutation, ${ }^{21}$ and 46 normal subjects from the general population in Spain. We also studied 12 patients with congenital unilateral absence of the vas deferens (CUAVD) and 10 patients with obstructive azoospermia not due to GBAVD or CUAVD. The patients with azoospermia but without CBAVD were in the care of the Andrology Department of the Institute of Urology, Nephrology, and Andrology in Barcelona, Spain, because of infertility; those with CUAVD were seen because of infertility or prostate problems or because they had requested vasectomy. The mean sperm concentration in the patients with CUAVD was $10.6 \times 10^{6}$ per milliliter (range, 0 to $90 \times 10^{6}$ ).

\section{Analysis of CFTR Mutations}

DNA was isolated from peripheral-blood lymphocytes according to standard protocols. ${ }^{22}$ Genomic DNA from the patients with CBAVD was first analyzed for the most common cystic fibrosis mutation, $\Delta \mathrm{F} 508 .^{5}$ To identify other cystic fibrosis mutations in these patients, each of the 27 exons of the CFTR gene and their flanking sequences were amplified by the polymerase chain reaction (PCR).

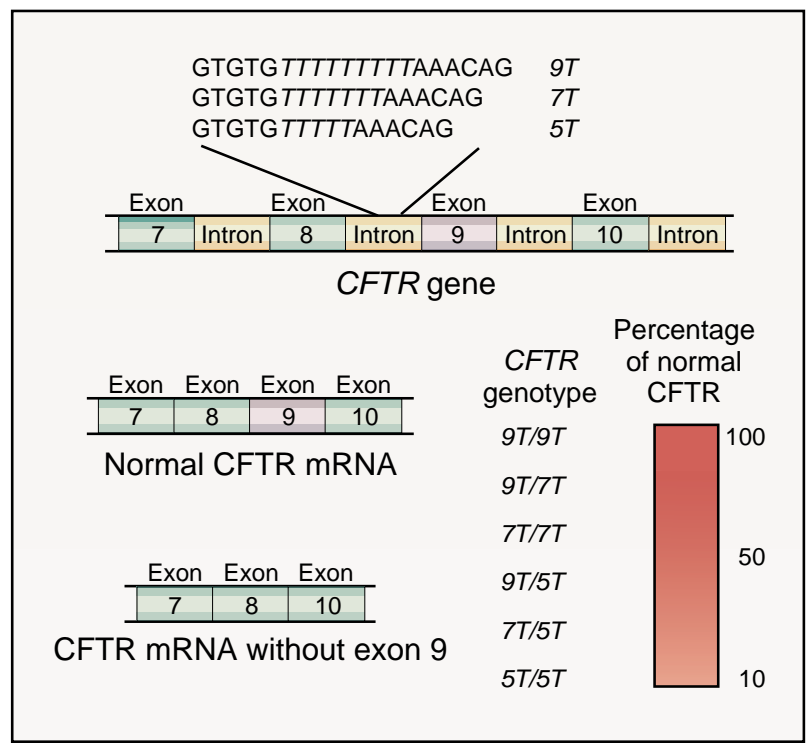

Figure 1. DNA Variants in Intron 8 of the CFTR Gene and Their Effects at the mRNA Level.

The region of the CFTR gene that includes exons 7 to 10 is shown at the top. During processing, the sequences not involved with protein synthesis (introns) are eliminated, and the remaining sequences (exons) are spliced to form mature mRNA (center left). The processing of CFTR is not completely efficient, because 10 to 92 percent of transcripts lack exon 9 (bottom left), depending on the person's genotype. ${ }^{13,18}$ When both CFTR genes bear the $5 T$ allele (the $5 T / 5 T$ genotype), the proportion of normal CFTR mRNA is reduced to approximately 8 to 12 percent, indicating that the shorter the sequence of thymines in intron 8 , the higher the proportion of CFTR mRNA in which exon 9 is lacking. ${ }^{18}$

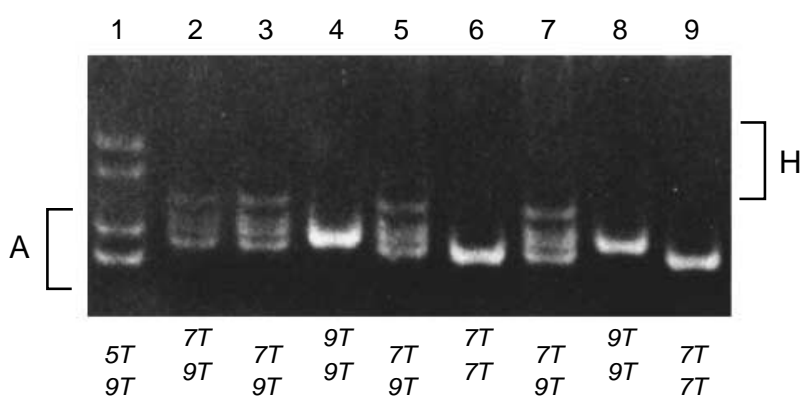

Figure 2. PCR Analysis of Alleles in the polyT Sequence of Intron 8 of the CFTR Gene.

Heteroduplex molecules $(\mathrm{H})$ are due to the hybridization of strands from the $5 T, 7 T$, and $9 T$ alleles (A). The genotypes are indicated beneath each lane.

After PCR, all exons were studied by denaturing gradient-gel electrophoresis or by single-strand conformation analysis, as previously described. ${ }^{23,24}$

\section{The $\mathbf{5 T}$ Allele of Intron 8 of CFTR}

We analyzed the frequency of the $5 T$ allele (the sequence of five thymines mainly responsible for the absence of exon 9 in CFTR mRNA) in the general population (i.e., in apparently normal chromosomes), fathers and mothers of patients with cystic fibrosis (who carry one chromosome with the cystic fibrosis mutation and one normal chromosome), and men with CBAVD. To evaluate the incidence of the $5 T$ allele of intron 8 in CBAVD and infertility, we studied the frequency of heterozygosity for the allele in patients with CBAVD, patients with CUAVD, patients with azoospermia but not CBAVD, and the general population.

Exon 9 was first amplified with primers 9 i-5 and 9 i- $3 .{ }^{25}$ The PCR conditions were as follows: denaturation at $95^{\circ} \mathrm{C}$ for 30 seconds, annealing at $54^{\circ} \mathrm{C}$ for 30 seconds, and extension at $74^{\circ} \mathrm{C}$ for 40 seconds, for 25 cycles. The reaction mixture contained $5 \mu$ l of PCR buffer (N808-0006, Perkin-Elmer Cetus); $200 \mu \mathrm{M}$ each of deoxyadenosine triphosphate, deoxycytidine triphosphate, deoxyguanosine triphosphate, and deoxythymidine triphosphate; 20 pmol of each primer; and 1 unit of Taq DNA polymerase in a final volume of $50 \mu$ l, containing $100 \mathrm{ng}$ of genomic DNA. To amplify the polypyrimidine sequence while avoiding the adjacent dinucleotide repeat $(\mathrm{GT}) \mathrm{n},{ }^{13}$ we performed a nested PCR with primers I9D9 (5'CGGCGGCTGTGTGTGTGTGTGTGTTTTT3') and E9R2 (5'GGATGCAGCAACGGCCAACA $\left.3^{\prime}\right)$. The conditions of the nested PCR were as described above except that $1 \mu \mathrm{l}$ from the first PCR was used, but for 35 cycles. The final PCR products were digested with $X m n \mathrm{I}$ and visualized on an 8 percent nondenaturing polyacrylamide gel after electrophoresis for four to five hours at $180 \mathrm{~V}$ (Fig. 2).

\section{Statistical Analysis}

Differences between proportions were tested by the chi-square statistic. ${ }^{26}$ Yates' correction for continuity was used in the two-by-two tables. Relative risks were calculated for the comparison of the patients with CBAVD with the normal patients. All $\mathrm{P}$ values were based on two-sided comparisons. $\mathrm{P}$ values of less than 0.05 were considered to indicate statistical significance.

\section{Results}

\section{CFTR Mutations in CBAVD}

We studied a group of 102 patients with CBAVD from Europe and the United States with regard to mutations in the CFTR gene. The analysis of the entire coding sequence allowed us to identify 28 different mutations (Table 1). Most of the mutations have been described previously in patients with cystic fibrosis, but 
Table 1. CFTR and polyT Genotypes of 102 Patients with CBAVD.

\begin{tabular}{|c|c|c|}
\hline CFTR GENOTYPE* & No. of Patients & POLYT GENOTYPE $\dagger$ \\
\hline$\Delta F 508 / R 668 C$ & 2 & $9 T / 7 T$ \\
\hline$\Delta F 508 / D 1152 H$ & 2 & $9 T / 7 T$ \\
\hline$\Delta F 508 / D 1270 N$ & 2 & $9 T / 7 T$ \\
\hline$\Delta F 508 / R 75 L$ & 1 & $9 T / 7 T$ \\
\hline$\Delta F 508 / R 117 H$ & 1 & $9 T / 7 T$ \\
\hline$\Delta F 508 / L 206 W$ & 1 & $9 T / 9 T$ \\
\hline$\Delta F 508 / R 258 G$ & 1 & $9 T / 7 T$ \\
\hline$\Delta F 508 / S 1235 R$ & 1 & $9 T / 7 T$ \\
\hline$\Delta F 508 / R 347 H$ & 1 & $9 T / 7 T$ \\
\hline$\Delta F 508 / R 347 H$ & 1 & $9 T / 9 T$ \\
\hline$R 117 H / G 1349 D$ & 1 & $7 T / 7 T$ \\
\hline$R 117 \mathrm{H} / 712-1 G \rightarrow T$ & 1 & $7 T / 9 T$ \\
\hline G149R/R668C & 1 & $9 T / 7 T$ \\
\hline R347H/R1066H & 1 & $9 T / 7 T$ \\
\hline R553X/R668C & 1 & $7 T / 7 T$ \\
\hline R1070W/2869insG & 1 & $7 T / 7 T$ \\
\hline$\Delta F 508 /-$ & 22 & $9 T / 5 T$ \\
\hline$G 542 X /-$ & 4 & $9 T / 5 T$ \\
\hline$W 1282 X /-$ & 3 & $7 T / 5 T$ \\
\hline$R 334 W /-$ & 1 & $7 T / 5 T$ \\
\hline K1060T/- & 1 & $7 T / 5 T$ \\
\hline$R 1162 X /-$ & 1 & $7 T / 5 T$ \\
\hline$N 1303 K /-$ & 1 & $9 T / 5 T$ \\
\hline A800G/- & 1 & $5 T / 5 T$ \\
\hline$\Delta F 508 /-$ & 7 & $9 T / 7 T$ \\
\hline$\Delta F 508 /-$ & 1 & $9 T / 9 T$ \\
\hline$\Delta F 508 /-$ & 1 & $7 T / 7 T$ \\
\hline$\Delta E 115 /-$ & 1 & $7 T / 7 T$ \\
\hline R117H/- & 1 & $7 T / 7 T$ \\
\hline R347H/- & 2 & $9 T / 7 T$ \\
\hline G542X/- & 1 & $9 T / 7 T$ \\
\hline$R 553 X /-$ & 1 & $7 T / 7 T$ \\
\hline 1677delTA/- & 1 & $7 T / 7 T$ \\
\hline 2184delA/- & 1 & $7 T / 7 T$ \\
\hline $2789+5 G \rightarrow A /-$ & 1 & $7 T / 7 T$ \\
\hline$S 1235 R /-$ & 1 & $7 T / 9 T$ \\
\hline$W 1282 X /-$ & 1 & $7 T / 7 T$ \\
\hline$-1-$ & 3 & $9 T / 5 T$ \\
\hline$-1-$ & 3 & $7 T / 5 T$ \\
\hline$-1-$ & 1 & $5 T / 5 T$ \\
\hline$-1-$ & 19 & $7 T / 7 T$ \\
\hline$-1-$ & 3 & $7 T / 9 T$ \\
\hline
\end{tabular}

*Minus signs indicate the absence of a CFTR mutation. $\dagger$ The $5 T$ allele is highlighted for greater visibility.

others have been detected specifically in patients with CBAVD. Nineteen patients had mutations in both copies of CFTR (one severe and one mild mutation in 16 patients, and two mild mutations in 3), and 54 patients had mutations in only one CFTR allele. In 29 patients, after comprehensive screening, we were unable to find any mutations in the coding or splice regions of CFTR. ${ }^{12,19,20}$

\section{Frequencies of the $5 T$ Allele DNA Variant of Intron 8 of CFTR}

In the Spanish population, the frequency of the $5 T$ allele, which is responsible for abnormal CFTR mRNA, was similar (5.4 percent) to that previously reported in other populations $(5.2 \text { percent })^{27-29}(\mathrm{P}=0.98)$, and the populations were pooled for comparative analyses (frequency of the $5 T$ allele in the general population, 5.2 percent). The frequency of the $5 T$ allele in the normal chromosomes of mothers of patients with cystic fibrosis (the non-cystic fibrosis chromosomes) was similar (4.5 percent) to that in the general population $(\mathrm{P}=0.87)$, but the frequency was lower in the normal chromosomes of fathers of patients with cystic fibrosis (2.1 percent) $(\mathrm{P}=0.12)$. In contrast, the $5 T$ allele was significantly more frequent in the chromosomes of patients with CBAVD (21.1 percent) than in the general population (chi-square $=39.3, \mathrm{P}<0.001)$ (Table 2 ).

\section{The $5 T$ Allele and Infertility}

We evaluated the incidence of the $5 T$ allele in men with various types of infertility. Table 3 shows that the percentage of patients with GBAVD who had this allele was significantly higher than that of the general population (40.2 vs. 10.9 percent) (chi-square $=11.4$, $\mathrm{P}<0.001$, relative risk $=5.1$ ), whereas the proportion of patients with CUAVD who had the $5 T$ allele (25 percent) was lower than, but not significantly different from, the proportion among patients with CBAVD $(\mathrm{P}=0.48)$. On the other hand, the proportion of patients with azoospermia but without CBAVD who had the $5 T$ allele was similar to that of the general population $(\mathrm{P}=0.71)$.

\section{CFTR Mutations and the 5 T Allele in Patients with CBAVD}

In most patients with CBAVD, the $5 T$ allele was strongly associated with the presence of a cystic fibrosis mutation in the other copy of the CFTR gene (chisquare $=9.9, \mathrm{P}=0.0016$ ), but none of the patients with CBAVD who had two CFTR mutations carried this allele (Table 4). Two patients were each found to have two $5 T$ alleles. In one patient one of the alleles was as-

Table 2. Frequencies of the polyT Alleles in Intron 8 of CFTR in Members of the General Population and Subjects with CBAVD, and in the Non-Cystic Fibrosis Chromosomes of Parents of Patients with Cystic Fibrosis.

\begin{tabular}{|c|c|c|c|}
\hline \multirow[t]{3}{*}{ Group StUdied } & \multicolumn{3}{|c|}{ POLyT Allele } \\
\hline & $5 T$ & $7 T$ & $9 T$ \\
\hline & \multicolumn{3}{|c|}{ no. with allele/no. studied (\%) } \\
\hline Spanish population* & $5 / 92 \quad(5.4)$ & $76 / 92 \quad(82.6)$ & $11 / 92 \quad(12.0)$ \\
\hline Other populations $\dagger$ & $21 / 406(5.2)$ & $339 / 406(83.5)$ & $46 / 406(11.3)$ \\
\hline General population $\ddagger$ & $26 / 498(5.2)$ & $415 / 498(83.3)$ & $57 / 498(11.5)$ \\
\hline Men with CBAVD & $43 / 204(21.1) \S$ & $97 / 204(47.5)$ & $64 / 204(31.4)$ \\
\hline \multicolumn{4}{|c|}{$\begin{array}{l}\text { Parents of patients with } \\
\text { cystic fibrosis }\end{array}$} \\
\hline Mothers & $2 / 44 \quad(4.5) \|$ & $39 / 44 \quad(88.6)$ & $3 / 44 \quad(6.8)$ \\
\hline Fathers & $4 / 186(2.1)^{* *}$ & $157 / 186(84.4)$ & $25 / 186(13.4)$ \\
\hline
\end{tabular}

*Data were obtained from the Spanish population analyzed in this study.

$\dagger$ Data were obtained from Kiesewetter et al., ${ }^{27}$ Dörk et al..${ }^{28}$ and Cuppens et al. ${ }^{29}$

$\ddagger$ Includes pooled data from the Spanish population studied and the studies listed above under "Other populations."

$\S \mathrm{P}<0.001$ for the comparison with the general population.

IFor these parents, data on the CFTR allele that was not transmitted to the patients with cystic fibrosis are shown. This allele is considered normal and is conventionally known as the noncystic fibrosis chromosome.

$\| \mathrm{P}=0.87$ for the comparison with the general population.

** $\mathrm{P}=0.12$ for the comparison with the general population 
Table 3. Frequency of Heterozygosity for the CFTR 5T Allele among Patients with CBAVD, CUAVD, or Azoospermia but No CBAVD and Members of the General Population.

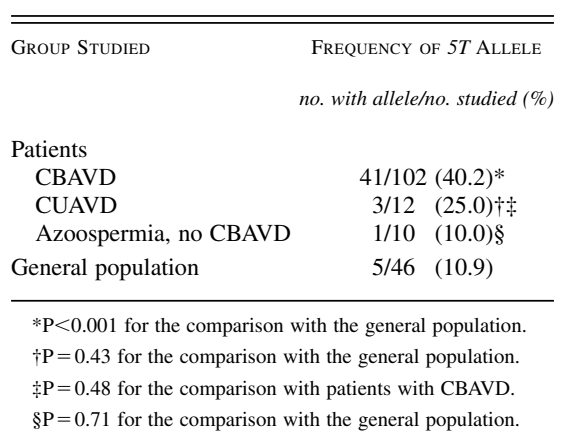

sociated with a mild cystic fibrosis mutation, ${ }^{19}$ whereas in the other patient no CFTR mutations were identified.

The association between the various CFTR mutations and the $5 T$ allele in the patients with CBAVD was analyzed by studying the transmission of the mutations within families (Table 1). Only one CFTR mutation (A800G) was associated with the $5 T$ allele, whereas all the others were associated with the $7 T$ or the $9 T$ allele, confirming that in each patient with CBAVD the $5 T$ allele corresponded to the chromosome that did not carry the $C F T R$ mutation.

\section{Discussion}

The main objectives of this study were to determine whether patients with CBAVD had mutations in the CFTR gene and to explore whether noncoding sequences that produce low levels of CFTR mRNA (the $5 T$ allele) were responsible for CBAVD.

Most patients with CBAVD in this study (72 percent) had a mutation in at least one of their CFTR genes, but only 19 percent had mutations on both chromosomes, with at least one of the two mutations being mild. ${ }^{12,19,20}$ Inability to identify the second mutation in most patients with CBAVD, even after all 27 CFTR exons were analyzed, suggests that mutations could be located elsewhere in the noncoding regions of CFTR. These mutations may result in a CFTR protein with a normal structure but low levels of expression, ${ }^{10}$ which may cause disease only in the organs most sensitive to CFTR dysfunction, such as the vas deferens. ${ }^{30,31}$

The reduced levels of normal GFTR mRNA due to the deletion of exon 9 depend on the presence of the $5 T$ allele sequence in intron 8. This nonfunctional CFTR mRNA accounts for up to 92 percent of the total mRNA when both CFTR genes have the $5 T$ allele. ${ }^{18}$ We have found a significant proportion of men with CBAVD who have the $5 T$ allele, as compared with men in the general population, which suggests that this allele functions as a disease mutation in CBAVD. Similarly, the proportion of men with CUAVD who have the $5 T$ allele was higher than in the general population, but lower than among men with CBAVD. Because CFTR mutations have also been found in patients with CUAVD, ${ }^{12,19}$ that condition could be an incomplete form of CBAVD. In contrast, the proportion of men with azoospermia but without CBAVD who had the $5 T$ allele was similar to that in the general population, suggesting that azoospermia not due to CBAVD or CUAVD is unrelated to CFTR.

The particular combination of the two CFTR alleles in a given person (the genotype) results in specific levels of normal CFTR mRNA and in a specific clinical phenotype (Fig. 3). It has been shown that if normal CFTR mRNA is present at a level of less than 1 to 3 percent, a severe cystic fibrosis phenotype results ${ }^{32}$; if the level is above 8 to 12 percent, the phenotype is normal $^{18}$; and at intermediate levels, the phenotype is one of mild cystic fibrosis. ${ }^{33}$ Thus, patients with one cystic fibrosis mutation on one chromosome and the $5 T$ allele on the other should have abnormally low levels of normal CFTR mRNA.

The study performed here allows patients with CBAVD to be classified in five categories (Table 4): patients with two CFTR mutations (group la, 19 percent of patients with GBAVD); patients with one CFTR mutation and the $5 T$ allele (group $1 \mathrm{~b}, 33$ percent); patients with only one CFTR mutation (group 2a, 20 percent); patients with only the $5 T$ allele (group 2b, 7 percent); and patients without $C F T R$ mutations (group 3, 21 percent). Group 1 is completely characterized if the $5 T$ allele is a mutation in patients with CBAVD, whereas in group 2 other, as yet unknown, mutations in the CFTR gene may be involved. Finally, in group 3, a gene or genes other than CFTR may be responsible for CBAVD.

Parents of patients with cystic fibrosis have one normal CFTR gene and one gene with a cystic fibrosis mutation. Since fathers of patients with cystic fibrosis are not infertile, if the $5 T$ allele was involved in CBAVD, it would be expected to be present at a low frequency in these subjects. Our data show that the frequency of the $5 T$ allele in fathers who carry the cystic fibrosis mutation is slightly lower than that in both the general population and mothers who carry the mutation (2.1 percent vs. 5.2 percent and 4.5 percent), reinforcing the hypothesis that the $5 T$ allele has a role in CBAVD (Table 2).

Four fathers who were carriers of cystic fibrosis had one CFTR gene with the $5 T$ allele and the other with a severe cystic fibrosis mutation (G542X, N1303K, 1812-1G $\rightarrow \mathrm{A}$, or 936delTA). Although these genotypes should have been associated with CBAVD, these men had offspring and are clinically normal. Three hypotheses could explain the strong but not complete correlation between the appearance of the $5 T$ allele and CBAVD. First, there could be a nonrandom association between the $5 T$ allele and CBAVD, with the allele segregating with the CBAVD phenotype but not being its cause. Second, there could be a partially causal role for the $5 T$ allele, together with additional mutations in other parts of the CFTR gene. Third, the $5 T$ allele could have a causal role in GBAVD, with other factors accounting for these exceptional men without CBAVD (the four fathers bearing the cystic fibrosis mutation). 
Table 4. Classification of 102 Patients with CBAVD According to the Presence or Absence of the CFTR Mutation and of a polyT Allele at Intron 8.

\begin{tabular}{cclc}
\hline \hline & $\begin{array}{c}\text { CFTR } \\
\text { Group }\end{array}$ & \multicolumn{1}{c}{$\begin{array}{c}\text { POLYT } \\
\text { GENOTYPE } \dagger\end{array}$} & $\begin{array}{c}\text { No. of } \\
\text { PATIENTS }(\%)\end{array}$ \\
$1 \mathrm{a}$ & $C F / C F$ & Non-5T/Non-5T & $19(18.6)$ \\
$\mathrm{1b}$ & $C F /-$ & Non-5T/5T $\neq$ & $34 \S(33.3)$ \\
$2 \mathrm{a}$ & $C F /-$ & Non-5T/Non-5T & $20(19.6)$ \\
$2 \mathrm{~b}$ & $-/-$ & Non-5T/5T $\neq$ & $7 \S(6.9)$ \\
3 & $-/-$ & Non-5T/Non-5T & $22(21.6)$ \\
\hline
\end{tabular}

*Minus signs indicate chromosomes with no mutations when the entire CFTR coding region was analyzed. $C F$ denotes cystic fibrosis mutation.

$\dagger N o n-5 T$ alleles denote either $7 T$ or $9 T$ alleles.

\$One patient in this group had the $5 T / 5 T$ genotype.

$\S$ The presence of the $5 T$ allele was strongly associated with heterozygosity for cystic fibrosis mutations $(\mathrm{P}=0.0016)$.

The work presented here argues convincingly against the first two hypotheses. Nonrandom association is not the case, since the analysis of several DNA markers within CFTR in the four fathers and in patients with CBAVD showed that several haplotypes (combinations of alleles on the same chromosome) were associated with the $5 T$ allele (data not shown). The presence of another mutation in the same CFTR gene as the $5 T$ allele is also excluded, since CFTR was thoroughly analyzed in all patients with CBAVD and it is extremely unlikely that all patients with the $5 T$ allele had mutations outside the CFTR coding region. These data and the association of the $5 T$ allele with low levels of normal CFTR mRNA ${ }^{18}$ strongly support the concept that the $5 T$ mutation generally causes CBAVD when it is associated with a cystic fibrosis mutation on the other chromosome.

Additional information about the importance of the $5 T$ mutation was obtained by screening 120 patients with cystic fibrosis. We identified three adults with the $\Delta F 508 / 5 T$ genotype who had mild lung disease starting in their 30s and CBAVD, but no pancreatic disease. Three other patients, 8,12 , and 14 years of age with the genotypes $E 585 X / 5 T$ and $K 710 X / 5 T$ (two were siblings), had a diagnosis of cystic fibrosis due to elevated concentrations of electrolytes in sweat $(>60$ mmol per liter) and episodes of dehydration, but no other clinical features. Since persons with a cystic fibrosis mutation and the $5 T$ allele may have levels of normal CFTR mRNA below the range of 8 to 12 percent (the minimal level for a normal phenotype ${ }^{18}$ ) but above the range of 1 to 3 percent (the level below which severe cystic fibrosis occurs ${ }^{31}$ ), a wide clinical variation is expected in them, depending on the variability of levels of normal CFTR mRNA. These clinical forms should include CBAVD, moderate cystic fibrosis, and the absence of fertility problems (Fig. 3).

In summary, we report the following findings: First, that the $5 T$ allele in intron 8 of CFTR has clinical effects related to male infertility. Second, that in 33 percent of cases the CBAVD phenotype results from the combined action of the $5 T$ allele and a cystic fibrosis mutation on the other chromosome. In addition, 19 percent of cases of CBAVD are due to the presence of two CFTR mutations other than the $5 T$ allele. Moreover, the presence of only one CFTR mutation (without the $5 T$ allele) in 20 percent of patients suggests that other undetected changes in CFTR may be involved in CBAVD. Furthermore, the relatively high proportion of patients with CBAVD who do not have CFTR mutations (22 percent) allows us to propose that another gene or genes could be responsible for CBAVD. Finally, CUAVD could be an incomplete form of CBAVD.

A large number of cystic fibrosis mutations have been discovered during the past five years, and it seems that we are now better prepared to understand how mutations combine to cause disease. The combination of the $5 T$ allele with a cystic fibrosis mutation in the other CFTR gene is the most common cause of CBAVD, but it also has other clinical presentations. Our report on CFTR mutations in patients with CBAVD indicates that CBAVD and cystic fibrosis are extreme forms of a wide

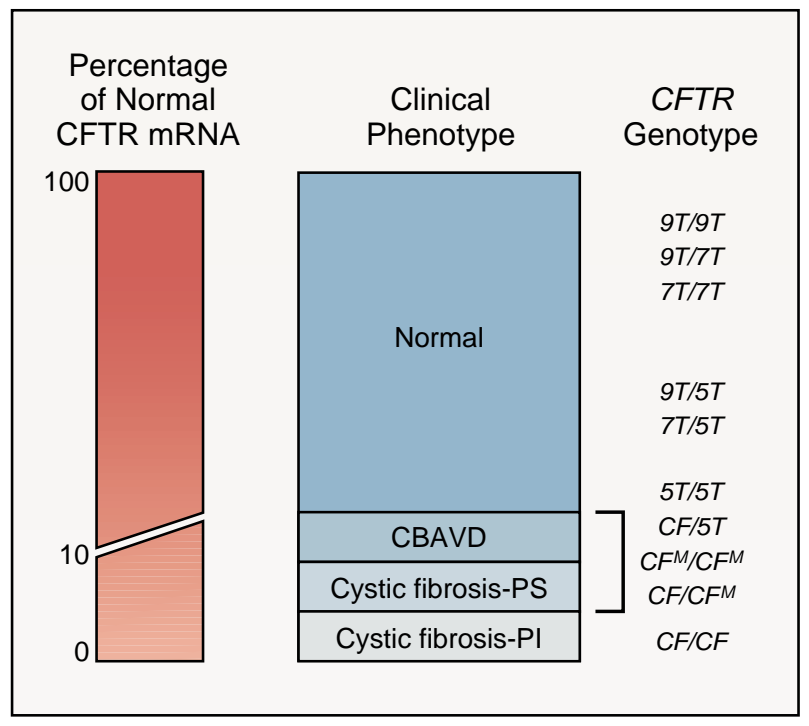

Figure 3. Comparison of Percentages of Normal CFTR mRNA, Clinical Phenotypes, and CFTR Genotypes.

Levels of normal CFTR mRNA depend on the genotype determining the length of the thymine sequence in intron 8 of CFTR, the presence of cystic fibrosis mutations, or both. Decreased levels of normal CFTR mRNA may be involved in various clinical phenotypes, ranging from the normal phenotype to the phenotypes of CBAVD, cystic fibrosis with pancreatic sufficiency (PS), and cystic fibrosis with pancreatic insufficiency (PI). Genotypes that correspond to the combination of a cystic fibrosis mutation with a $5 T$ allele $(C F / 5 T)$ have been found in normal persons, patients with CBAVD, and patients with cystic fibrosis and pancreatic sufficiency. Genotypes combining a severe and a moderate cystic fibrosis mutation $\left(C F / C F^{M}\right)$ or two moderate mutations $\left(C^{M} / C F^{M}\right)$ can be involved in either CBAVD or cystic fibrosis with pancreatic sufficiency (bracket). The delimitation between the normal, CBAVD, and cystic fibrosis phenotypes and their relations with levels of CFTR mRNA is only approximate. The distribution of levels of CFTR mRNA in relation to the presence of the $5 T, 7 T$, and $9 T$ alleles and genotypes is derived from the work of Chu et al. ${ }^{18}$ 
nosologic spectrum of conditions that have a common molecular basis.

We are indebted to Drs. M. Pritchard and F. Cardellach for useful suggestions and comments, to $\mathrm{H}$. Kruyer for assistance with the manuscript, and to J. Giménez, M.D. Ramos, and M. Miranda for technical assistance.

\section{REFERENCES}

1. Jequier AM, Ansell ID, Bullimore NJ. Congenital absence of the vasa deferentia presenting with infertility. J Androl 1985;6:15-9.

2. Boat TF, Welsh MJ, Beaudet AL. Cystic fibrosis. In: Scriver CR, Beaudet AL, Sly WS, Valle D, eds. The metabolic basis of inherited disease. 6th ed. Vol. 2. New York: McGraw-Hill, 1989:2649-80.

3. Riordan JR, Rommens JM, Kerem B, et al. Identification of the cystic fibrosis gene: cloning and characterization of complementary DNA. Science 1989;245:1066-73. [Erratum, Science 1989;245:1437.]

4. Anderson MP, Berger HA, Rich DP, Gregory RJ, Smith AE, Welsh MJ. Nucleoside triphosphates are required to open the CFTR chloride channel. Cell 1991;67:775-84.

5. Kerem BS, Rommens JM, Buchanan JA, et al. Identification of the cystic fibrosis gene: genetic analysis. Science 1989;245:1073-80.

6. Tsui L-C. The spectrum of cystic fibrosis mutations. Trends Genet 1992;8: 392-8.

7. The Cystic Fibrosis Genotype-Phenotype Consortium. Correlation between genotype and phenotype in patients with cystic fibrosis. N Engl J Med 1993; 329:1308-13.

8. Dumur V, Gervais R, Rigot JM, et al. Abnormal distribution of CF $\Delta \mathrm{F} 508$ allele in azoospermic men with congenital aplasia of epididymis and vas deferens. Lancet 1990;336:512.

9. Anguiano A, Oates RD, Amos JA, et al. Congenital bilateral absence of the vas deferens: a primary genital form of cystic fibrosis. JAMA 1992;267: 1794-7.

10. Osborne LR, Lynch M, Middleton PG, et al. Nasal epithelial ion transport and genetic analysis of infertile men with congenital bilateral absence of the vas deferens. Hum Mol Genet 1993;2:1605-9. [Erratum, Hum Mol Genet 1993;2:1990.]

11. Gervais R, Dumur V, Rigot JM, et al. High frequency of the R117H cystic fibrosis mutation in patients with congenital absence of the vas deferens. N Engl J Med 1993;328:446-7.

12. Culard JF, Desgeorges M, Costa P, et al. Analysis of the whole CFTR coding regions and splice junctions in azoospermic men with congenital bilateral aplasia of epididymis or vas deferens. Hum Genet 1994;93:467-70.

13. Chu CS, Trapnell BC, Murtagh JJ Jr, et al. Variable deletion of exon 9 coding sequences in cystic fibrosis transmembrane conductance regulator gene mRNA transcripts in normal bronchial epithelium. EMBO J 1991;10:1355-63.

14. Bremer S, Hoof T, Wilke M, et al. Quantitative expression patterns of multidrug-resistance P-glycoprotein (MDR1) and differentially spliced cysticfibrosis transmembrane-conductance regulator mRNA transcripts in human epithelia. Eur J Biochem 1992;206:137-49.

15. Slomski R, Schloesser M, Berg LP, et al. Omission of exon 12 in cystic fibrosis transmembrane conductance regulator (CFTR) gene transcripts. Hum Genet 1992;89:615-9.
16. Delaney SJ, Rich DP, Thomson SA, et al. Cystic fibrosis transmembrane conductance regulator splice variants are not conserved and fail to produce chloride channels. Nat Genet 1993;4:426-31.

17. Strong TV, Wilkinson DJ, Mansoura MK, et al. Expression of an abundant alternatively spliced form of the cystic fibrosis transmembrane conductance regulator (CFTR) gene is not associated with a cAMP-activated chloride conductance. Hum Mol Genet 1993;2:225-30.

18. Chu CS, Trapnell BC, Curristin S, Cutting GR, Crystal RG. Genetic basis of variable exon 9 skipping in cystic fibrosis transmembrane conductance regulator mRNA. Nat Genet 1993;3:151-6.

19. Casals T, Bassas LL, Ruiz-Romero J, et al. Extensive analysis of 40 infertile patients with congenital absence of the vas deferens: in $50 \%$ of cases only one CFTR allele could be detected. Hum Genet 1995;95:205-11.

20. Mercier B, Verlingue $\mathrm{C}$, Lissens W, et al. Is congenital bilateral absence of vas deferens a primary form of cystic fibrosis? Analyses of the CFTR gene in 67 patients. Am J Hum Genet 1995;56:272-7.

21. Chillón M, Casals T, Giménez J, et al. Analysis of the CFTR gene confirms the high genetic heterogeneity of the Spanish population: 43 mutations account for only $78 \%$ of CF chromosomes. Hum Genet 1994;93:447-51.

22. Miller SA, Dykes DD, Polesky HF. A simple salting out procedure for extracting DNA from human nucleated cells. Nucleic Acids Res 1988;16:1215.

23. Audrézet MP, Mercier B, Guillermit H, et al. Identification of 12 novel mutations in the CFTR gene. Hum Mol Genet 1993;2:51-4. [Erratum, Hum Mol Genet 1993;2:496.]

24. Chillón M, Casals T, Giménez J, Nunes V, Estivill X. Analysis of the CFTR gene in the Spanish population: SSCP-screening for 60 known mutations and identification of four new mutations (Q30X, A120T, 1812-1 G $\rightarrow$ A, and 3667del4). Hum Mutat 1994;3:223-30.

25. Zielenski J, Rozmahel R, Bozon D, et al. Genomic DNA sequence of the cystic fibrosis transmembrane regulator (CFTR) gene. Genomics 1991;10: 214-28.

26. Fleiss JL. Statistical methods for rates and proportions. 2nd ed. New York: John Wiley, 1981

27. Kiesewetter S, Macek M Jr, Davis C, et al. A mutation in CFTR produces different phenotypes depending on chromosomal background. Nat Genet 1993;5:274-8.

28. Dörk T, Fislage R, Neumann T, Wulf B, Tümmler B. Exon 9 of the CFTR gene: splice site haplotypes and cystic fibrosis mutations. Hum Genet 1994; 93:67-73

29. Cuppens H, Teng H, Raeymaekers P, De Boeck C, Cassiman JJ. CFTR haplotype backgrounds on normal and mutant CFTR genes. Hum Mol Genet 1994;3:607-14

30. Trezise AE, Chambers JA, Wardle CJ, Gould S, Harris A. Expression of cystic fibrosis gene in human foetal tissues. Hum Mol Genet 1993;2:213-8.

31. Tizzano EF, Chitayat D, Buchwald M. Cell-specific localization of CFTR mRNA shows developmentally regulated expression in human fetal tissues. Hum Mol Genet 1993;2:219-24.

32. Chillón M, Dörk T, Casals T, et al. A novel donor splice site in intron 11 of the CFTR gene, created by mutation $1811+1.6 \mathrm{kbA} \rightarrow \mathrm{G}$, produces a new exon: high frequency in Spanish cystic fibrosis chromosomes and association with severe phenotype. Am J Hum Genet 1995;56:623-9.

33. Highsmith WE, Burch LH, Zhou Z, et al. A novel mutation in the cystic fibrosis gene in patients with pulmonary disease but normal sweat chloride concentrations. N Engl J Med 1994;331:974-80. 